\title{
Magnetic Resonance Imaging of the Temporomandibular Joint at 7.0 T Using High-Permittivity Dielectric Pads: A Feasibility Study
}

\author{
Manoliu, Andrei ; Spinner, Georg ; Wyss, Michael ; Ettlin, Dominik A ; Nanz, Daniel ; Kuhn, Felix P ;
} Gallo, Luigi M ; Andreisek, Gustav

\begin{abstract}
OBJECTIVES The aims of this study were to show feasibility and to quantitatively and qualitatively evaluate the use of high-permittivity dielectric pads for imaging the temporomandibular joint (TMJ) at 7.0 T. MATERIALS AND METHODS This study is an institutional review board-approved study with written informed consent. Ten asymptomatic volunteers (20 TMJs) were magnetic resonance imaged using a 32-channel head coil at 7.0 T (Achieva; Philips Healthcare, the Netherlands) with and without high-permittivity dielectric pads consisting of barium titanate in deuterated suspension. Imaging protocol consisted of an oblique sagittal proton density-weighted turbo-spin echo sequence. For quantitative evaluation, B1 maps and voxelwise signal-to-noise ratio (SNR) maps were calculated. For qualitative evaluation, 2 readers assessed the visibility of anatomical structures of the TMJ and overall image quality on a 5-point Likert scale from 1 (excellent visibility) to 5 (not visible) in consensus. Quantitative and qualitative measurements were compared between images acquired with and without pads. RESULTS Imaging the TMJ using dielectric pads was feasible in all volunteers. The quantitative analysis showed locally higher B1+ and higher SNR in the area covering the TMJ for the scans performed with dielectric pads compared with those without pads (SNR: mean [SD] pads, 12.38 [3.18]; mean [SD] no pads, 6.60 $[0.72])$. The qualitative analysis showed significantly better visibility and delineation of clinically relevant anatomical structures of the TMJ, including temporomandibular disc, bilaminar zone, mandibular fossa, mandibular condyle, and pterygoid muscle. In addition, observers judged overall image quality as better for images taken with pads compared with those taken without pads (mean [SD] pads, 1.40 [0.50]; mean [SD] no pads, 4.25 [0.78]). CONCLUSIONS The application of high-permittivity dielectric pads improves the local B1+ field and thus the SNR, optimizing TMJ magnetic resonance imaging at 7.0 T.
\end{abstract}

DOI: https://doi.org/10.1097/RLI.0000000000000196

Posted at the Zurich Open Repository and Archive, University of Zurich

ZORA URL: https://doi.org/10.5167/uzh-112353

Journal Article

Published Version

Originally published at:

Manoliu, Andrei; Spinner, Georg; Wyss, Michael; Ettlin, Dominik A; Nanz, Daniel; Kuhn, Felix P; Gallo, Luigi M; Andreisek, Gustav (2015). Magnetic Resonance Imaging of the Temporomandibular Joint at 7.0 T Using High-Permittivity Dielectric Pads: A Feasibility Study. Investigative Radiology, 50(12):843-849. DOI: https://doi.org/10.1097/RLI.0000000000000196 


\title{
Magnetic Resonance Imaging of the Temporomandibular Joint at 7.0 T Using High-Permittivity Dielectric Pads A Feasibility Study
}

\author{
Andrei Manoliu, MD, PhD, *† Georg Spinner, MSc, $\uparrow$ Michael Wyss, BSc, $†$ Dominik A. Ettlin, MD, DMD, $\neq$ \\ Daniel Nanz, PhD, * Felix P. Kuhn, MD, MAS, * Luigi M. Gallo, PhD, $\neq$ and Gustav Andreisek, MD, MBA*
}

\begin{abstract}
Objectives: The aims of this study were to show feasibility and to quantitatively and qualitatively evaluate the use of high-permittivity dielectric pads for imaging the temporomandibular joint (TMJ) at $7.0 \mathrm{~T}$.

Materials and Methods: This study is an institutional review board-approved study with written informed consent. Ten asymptomatic volunteers (20 TMJs) were magnetic resonance imaged using a 32-channel head coil at 7.0 T (Achieva; Philips Healthcare, the Netherlands) with and without high-permittivity dielectric pads consisting of barium titanate in deuterated suspension. Imaging protocol consisted of an oblique sagittal proton density-weighted turbo-spin echo sequence. For quantitative evaluation, B1 maps and voxelwise signal-to-noise ratio (SNR) maps were calculated. For qualitative evaluation, 2 readers assessed the visibility of anatomical structures of the TMJ and overall image quality on a 5-point Likert scale from 1 (excellent visibility) to 5 (not visible) in consensus. Quantitative and qualitative measurements were compared between images acquired with and without pads.

Results: Imaging the TMJ using dielectric pads was feasible in all volunteers. The quantitative analysis showed locally higher B1+ and higher SNR in the area covering the TMJ for the scans performed with dielectric pads compared with those without pads (SNR: mean [SD] pads, 12.38 [3.18]; mean [SD] no pads, 6.60 [0.72]). The qualitative analysis showed significantly better visibility and delineation of clinically relevant anatomical structures of the TMJ, including temporomandibular disc, bilaminar zone, mandibular fossa, mandibular condyle, and pterygoid muscle. In addition, observers judged overall image quality as better for images taken with pads compared with those taken without pads (mean [SD] pads, 1.40 [0.50]; mean [SD] no pads, 4.25 [0.78]).

Conclusions: The application of high-permittivity dielectric pads improves the local B1+ field and thus the SNR, optimizing TMJ magnetic resonance imaging at $7.0 \mathrm{~T}$.
\end{abstract}

Key Words: temporomandibular joint, ultrahigh magnetic field strengths, high-permittivity dielectric pads

(Invest Radiol 2015;50: 843-849)

$\mathrm{T}$ emporomandibular disorders (TMDs) is a collective term for various pathologies of the temporomandibular joint (TMJ) and surrounding tissues, ${ }^{1}$ which are characterized by common features, including

Received for publication April 16, 2015; and accepted for publication, after revision, May 29, 2015.

From the *Department of Radiology, Institute for Diagnostic and Interventional Radiology, University Hospital Zurich, University of Zurich; $\dagger$ Institute for Biomedical Engineering, University of Zurich and ETH Zurich; and †Center of Dental Medicine, University of Zurich, Zurich, Switzerland.

This study is a prospective institutional review board-approved magnetic resonance imaging study. It included imaging of asymptomatic healthy volunteers. All volunteers gave written informed consent.

Conflicts of interest and sources of funding: this study was funded by the Swiss National Science Foundation grant number 320030 156466. No industry support was received for this study. G.A. receives grants from Siemens Healthcare. For the remaining authors, none were declared.

Correspondence to: Andrei Manoliu, MD, PhD, Department of Radiology, Institute for Diagnostic and Interventional Radiology, University Hospital Zurich, Ramistrasse 100,8091 Zurich, Switzerland. E-mail: andrei.manoliu@usz.ch.

Copyright (c) 2015 Wolters Kluwer Health, Inc. All rights reserved.

ISSN: 0020-9996/15/5012-0843

DOI: $10.1097 /$ RLI.0000000000000196 pain, clicking or crepitus, and alterations of the mouth-opening path. ${ }^{2}$ Although TMDs often compromise the quality of life $^{3}$ and cause great socioeconomic costs, ${ }^{4}$ the diagnostic process is still subject of considerable debate and requires optimization. ${ }^{5}$

Over the last decades, magnetic resonance imaging (MRI) arose as the leading imaging method to assess TMJ conditions. ${ }^{6}$ Currently, clinical MRI is performed at 1.5 and 3.0 T. ${ }^{7-9}$ However, the correlation between image findings and clinical symptoms in patients with TMDs is still unsatisfying, suggesting that imaging methods still need further improvement to adequately depict the fine anatomical structures of the TMJ, such as the articular disc. ${ }^{10}$ One approach to increase the performance of MRI is to increase the signal-to-noise ratio (SNR), which is assumed to scale roughly linearly with the field strength. Therefore, MRI of the TMJ at field strengths such as 7.0 T can be expected to enhance the visibility and delineation of clinically relevant structures compared with 1.5 or $3.0 \mathrm{~T} .{ }^{11}$ However, the TMJ has not yet been imaged at 7.0 T due to significant challenges, such as strong local inhomogeneities in the transmit radiofrequency field (RF; $\mathrm{B} 1+$ ) when imaging the head at 7.0 T, which lead to a heterogeneous distribution of flip angles with larger flip angles at the center of the head and smaller flip angels at the lateral regions of the head, particularly in the regions where the TMJ is located. ${ }^{12}$

Recently, it has been demonstrated that dielectric pads consisting of suspended metal titanates can modulate the B1+ field without increasing the specific absorption rate (SAR). ${ }^{13}$ To use this effect for imaging the inner ear at 7.0 T, Brink and colleagues ${ }^{14}$ manufactured high-permittivity dielectric pads consisting of barium titanate suspended in deuterated water, which account for the local constraints of close-fitting coils. They found that the dielectric pads locally increased the $\mathrm{B} 1+$ field in the region covering the inner ear at the cost of global $\mathrm{B} 1+{ }^{14}$ and therefore enabled MRI of the fine structures of the inner ear at 7.0 $\mathrm{T}$ at very high-spatial resolution. We hypothesize that the application of high-permittivity dielectric pads should increase the local $\mathrm{B} 1+$ in the location of the TMJ, yielding both higher SNR and better visualization of the anatomical subregions of the TMJ at 7.0 T.

Thus, the aim of the current study was to quantitatively and qualitatively evaluate the application of dielectric pads for detailed imaging of clinically relevant TMJ structures at 7.0 T.

\section{MATERIALS AND METHODS}

This prospective MRI study in asymptomatic volunteers was approved by the local institutional review board. All participants gave written informed consent. The study was registered in the official research data base of the University of Zurich. It was funded by the Swiss National Science Foundation (reference number 320030_156466/1).

\section{Subjects}

Ten healthy asymptomatic volunteers were consecutively included in this study ( 5 women [mean age, 24.2 years; range, 19-29 years] and 5 men [mean age, 26.7 years; range, 21-32 years). Inclusion criteria were absence of history or symptoms related to TMD. Exclusion criteria were pregnancy, metallic implants (including retainers), and claustrophobia. No contrast agent was administered in this study. 


\section{Dielectric Pads}

Dielectric pads specifically tailored to improve the local B1+ field in the lateral areas of the head were used in this study. Pads were manufactured using a suspension of barium titanate (325 mesh powder; Alfa Aesar GmbH \& Co KG, Karlsruhe, Germany) and deuterated water (99.9\%, Sigma Aldrich, Zwijndrecht, the Netherlands), which yielded a relative permittivity of 286 and an electrical conductivity of $0.44 \mathrm{~S} / \mathrm{m}$ at $298 \mathrm{MHz}$. The geometry of the manufactured pads was based on simulations performed by Brink and colleagues, ${ }^{14}$ and resulted in 2 different sets of dielectric pads for male and female subjects to account for sex-specific differences in head size. The pads for imaging females (right pad, $140 \times 140 \times 10 \mathrm{~mm}^{3}$; left pad, $100 \times 100 \times$ $10 \mathrm{~mm}^{3}$; see Fig. 1A) were smaller than those for male volunteers (right pad, $180 \times 140 \times 10 \mathrm{~mm}^{3}$; left pad, $100 \times 100 \times 10 \mathrm{~mm}^{3}$; see Fig. 1B).

\section{Magnetic Resonance Imaging}

\section{Imaging Protocol}

Magnetic resonance imaging was performed on a 7.0 T Philips Achieva system (Philips Healthcare, Cleveland, $\mathrm{OH}$ ) using a quadrature transmit head coil in combination with a 32-channel receive array (NOVA Medical, Wilmington, MA). Proton density-weighted fast spin echo sequences in oblique sagittal planes were acquired using the following sequence parameters: repetition time, 3300 milliseconds; echo time (TE), 22 milliseconds; flip angles (excitation, 90 degrees; refocusing, 100 degrees); echo train length, 84 milliseconds; inter-TE, 12 milliseconds; effective TE, 21 milliseconds; field of view (FOV), $150 \times 150 \mathrm{~mm}^{2}$; pixel size, $0.4 \times 0.4 \mathrm{~mm}^{2}$; reconstructed pixel size, $0.2 \times 0.2 \mathrm{~mm}^{2}$; slice thickness, $2 \mathrm{~mm}$; number of stacks, 2 (each stack covering $1 \mathrm{TMJ}$, respectively); number of slices per stack, 12 (resulting in a total of 24 slices covering both TMJs); number of signal averages, 1; and total acquisition time, 5:49 minutes. For reconstruction, vendor-specific algorithms were used.

\section{Noise Scans and B1 Mapping}

Image acquisition was accompanied by an identical scan without RF excitation and gradient switching to assess noise and allow SNR calculations on a voxelwise basis. Before B1 mapping, thirdorder shimming of the B0 field was performed. B1 mapping was performed by acquiring a slab of 10 slices at a spatial resolution of $3.0 \times$ $3.0 \times 8.0 \mathrm{~mm}^{3}$ using the actual flip angle method. ${ }^{15}$ This gradient echo-based sequence consisted of a constant flip angle and 2 interleaved different repetition times. The pixel value in the calculated B1 image was scaled as a percentage of the nominal transmit RF amplitude. A pixel value of $100(\%)$ meant that the RF power optimization preparation phase had adjusted RF power such that all RF pulses had exactly their nominal flip angles. It has to be noted that the accuracy of measured B1+ values was limited by the MR signal dropout caused by the temporal bone. Nevertheless, sufficient B1+ signal could be measured in nearby tissue (see Fig. 2B for detailed presentation of the region of interest).

\section{Subject Imaging}

All subjects were scanned successively with and without pads, resulting in 2 imaging sessions for all volunteers (mean [SD] time between sessions, 3.10 [1.14] minutes).

For MRI with pads, both pads were centered on the TMJs of each subject. To avoid potential bias due to position and scanner drift, half of the participants $(n=5)$ were first imaged using the dielectric pads. The other half of the participants $(n=5)$ were first imaged without dielectric pads. Care was taken to ensure correct and identical positioning of the participants throughout the study. Therefore, the first author of this study was present at all scans and confirmed the correct positioning (Fig. 1C). Magnetic resonance images of both TMJs were taken with closed mouth. The FOV was always positioned perpendicularly to the condylar main axis. Both TMJs were imaged simultaneously.

\section{Data Analysis}

\section{B1+ Measurements}

The effect of dielectric pads has been fully described by Brink and colleagues. ${ }^{14}$ To reproduce the potential effect of dielectric pads in the context of the current study, B1+ values were extracted using MIPAV (http://mipav.cit.nih.gov) for every B1+ map along a profile line. In particular, the white line starts on the level of the margin of the right TMJ $(0 \mathrm{~mm})$ and ends on the level of the margin of the left TMJ (94 mm). It is to note that accuracy of measured B1+ values was limited by the MR signal dropout caused by the temporal bone. However, sufficient B1 signal could be extracted from surrounding tissue. ${ }^{14}$

\section{SNR Measurements}

For SNR analysis, data were examined on a voxelwise basis, which allowed the calculation of SNR maps within the whole FOV. Image and corresponding noise data of every coil channel were postprocessed using dedicated software routines (Matlab, Natick, MA) yielding voxel-based SNR maps. ${ }^{11}$ In particular, SNR was calculated as described in full detail by Nordmeyer-Massner and colleagues ${ }^{16}$ :

$$
\mathrm{SNR}=\frac{|\rho|}{\sigma} \approx \sqrt{d^{\mathrm{H}} \psi^{-1} d}
$$

where $\rho$ is the exact magnitude of the available transverse magnetization at the voxel position according to Roemer et $a 1,{ }^{17} \sigma$ is the standard deviation of its noise components according to Pruessmann et $\mathrm{al}^{18} d$ lists the complex values that a given pixel exhibits in the


FIGURE 1. High-permittivity dielectric pads. Based on simulations performed by Brink and colleagues, ${ }^{14} 2$ different sets of dielectric pads for male and female subjects were manufactured to account for sex-specific differences in head size. A, The set for female volunteers (right pad, $140 \times 140 \times 10 \mathrm{~mm}^{3}$; left pad, $100 \times 100 \times 10 \mathrm{~mm}^{3}$ ). B, The set for male volunteers (right pad, $180 \times 140 \times 10 \mathrm{~mm}^{3}$; left pad, $100 \times 100 \times 10 \mathrm{~mm}^{3}$ ). C, The placement of the pads, which was performed by centering both pads on the TMJ of each subject as an anatomical landmark. Figure 1 can be viewed online in color at www.investigativeradiology.com. 


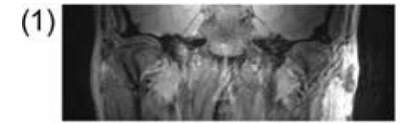

(2)

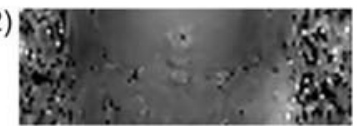

(3)

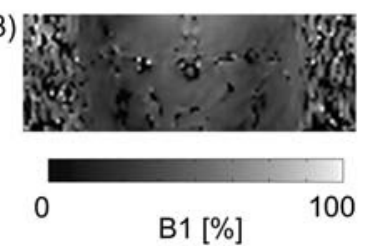

A



B



C

$[\mathrm{mm}]$

FIGURE 2. B1+ map of a representative volunteer acquired with and without dielectric pads. A1, The image presents an anatomical reference image in coronal orientation imaged using high-permittivity dielectric pads. Panel A2 shows the B1+ distribution with dielectric pads, whereas panel A3 shows the B1 distribution without dielectric pads for the same volunteer. The B1 image is scaled as a percentage of nominal transmit RF amplitude. A pixel value of $100 \%$ means that the RF power optimization preparation phase has adjusted RF power such that all RF pulses have exactly their nominal flip angles. $\mathrm{B} 1+$ values are gray scale-coded from 0 (black) to 100 (white). B, The area from which B1 has been extracted for further evaluation (white line). It is to note that accuracy of measured B1+ values was limited by the MR signal dropout caused by the temporal bone. However, sufficient B1 signal could be extracted from surrounding tissue. In particular, the white line starts on the level of the margin of the right TMJ ( 0 mm) and ends on the level of the margin of the left TMJ $(94 \mathrm{~mm})$. C, A profile plot along a the aforementioned line from a B1 map with and without pads for a representative volunteer, whereas the blue line indicates B1 with dielectric pads and the red line indicates B1 without dielectric pads. It is to note that using dielectric pads, lateral regions yielded increased B1, whereas B1 in the center of the head decreased. Figure 2 can be viewed online in color at www.investigativeradiology.com.

different single-channel images, the superscript $\mathrm{H}$ denotes the complex conjugate transpose, and $\psi$ denotes the noise covariance matrix calculated from the noise scans according to Pruessmann et al. ${ }^{18}$ Subsequently, each TMJ disc, fossa, and condyle were manually segmented by 1 author (blinded for review) for each SNR map. Corresponding SNR values were extracted, resulting in $1 \mathrm{SNR}$ value for each subject's TMJ.

\section{Qualitative Image Evaluation}

All images were transferred to the hospital picture archiving and communication system (Impax 6.0; Agfa Healthcare, Mortsel, Belgium) and evaluated by 2 fellowship-trained radiologists in consensus (GA) and (AM), 14 and 4 years of experience, respectively, with MRI of the musculoskeletal system). All images were anonymized (subject's initials blinded) and evaluated in random order. They rated the overall image quality and the visibility of clinically relevant structures, including the articular disc, the bilaminar zone, the mandibular fossa, the mandibular condyle, and the inferior lateral pterygoid muscle. In particular, the articular disc was evaluated with respect to anatomical subregions (anterior band, intermediate zone, posterior band). According to a previously reported grading system, ${ }^{8}$ the accuracy of representation of anatomical structures was rated on a Likert scale from 1 to 5 (1, excellent visibility; 2 , good visibility; 3 , moderate visibility; 4 , poor visibility; 5 , not visible).

\section{Statistical Analysis}

Normal distribution of the SNR was evaluated using the ShapiroWilk test (significance level $\alpha=0.05$ ). Paired sample $t$ tests were used to evaluate for statistically significant SNR differences between the images acquired with and without pads as well as between measured SNR on the left and right side when using dielectric pads. Two-sample $t$ tests were used to assess between-group differences between images from female and male volunteers with and without dielectric pads. To investigate statistically significant between-group differences between the images acquired with and without pads with respect to the visibility of clinically relevant anatomical structures, respectively, Wilcoxon signed rank tests were performed (significance level $\alpha=0.05$, corrected for multiple comparisons [ $\mathrm{n}=8$ according to the number of evaluated anatomical structures]). Furthermore, Wilcoxon signed rank tests were performed to evaluate potential differences between the left and the right TMJ when using dielectric pads significance level $\alpha=0.05$, corrected for multiple comparisons. To test for potential differences between sex groups regarding the visibility of the TMJ's subregions, Mann-Whitney $U$ tests were performed (significance level $\alpha=0.05$, corrected for multiple comparisons $[\mathrm{n}=8]$ ). All statistical analyses were performed using SPSS (release 22.0; SPSS Inc, Chicago, IL).

\section{RESULTS}

\section{Quantitative Analysis}

In images obtained without pads, the areas of low B1+ were overlapping with the TMJ causing low SNR (see Fig. 2A). In contrast, dielectric pads improved the local $\mathrm{B} 1+$ field, which translated into higher SNR in the region of interest, yielding an improved image quality (see below). Figure 2C demonstrates the effect of the dielectric pads on the $\mathrm{B} 1+$ field in a representative asymptomatic subject.

Signal-to-noise ratio was normally distributed with and without pads (pads, $P=0.315$; no pads, $P=0.752$ ). Quantitative SNR analysis within the area overlapping with the TMJ revealed significantly higher SNR for the images acquired with pads compared with those acquired without pads (mean [SD] pads, 12.38 [3.18]; mean [SD] no pads, 6.60 [0.72]; $t=7.760 ; P<0.001$; see Fig. 3). Signal-to-noise ratio was not significantly different between the left and right side with pads (mean [SD] left, 12.86 [3.48]; mean [SD] right, 11.90 [2.94]; $t=1.997$; $P=0.77$ ) as well as without pads (mean [SD] left, 6.82 [0.89]; mean [SD] right, 6.39 [0.43]; $t=1.946 ; P=0.84)$.

Furthermore, there was no statistical difference regarding SNR for female and male subjects with pads (mean [SD] females, 13.80 [3.62]; mean [SD] males, $12.04[3.06] ; t=0.83 ; P=0.43)$ as well as without pads (mean [SD] females, 6.54 [0.957]; mean [SD] males, $6.73[0.350] ; t=-0.42 ; P=0.158)$

\section{Qualitative Assessment}

Twenty TMJ images of 10 subjects were evaluated (see Table 1 for mean [SD] for the visibility of analyzed TMJ structures in images acquired with and without dielectric pads). The use of dielectric pads resulted in significantly improved overall image quality $(P=0.003$, corrected for multiple comparisons). In addition, images acquired with 




A



B
Without Pads



FIGURE 3. Voxelwise SNR maps for images acquired with and without pads. For SNR analysis, data were examined on a voxelwise basis. Image data and corresponding noise data of every coil channel were postprocessed individually using dedicated software routines, yielding voxel-based SNR maps. A, The voxelwise SNR map for a representative volunteer with dielectric pads. B, The voxelwise SNR map for the same TMJ of the same volunteer without dielectric pads. Signal-to-noise ratio was significantly higher within the region of interest (articular disc, fossa, and condyle) when using dielectric pads. Signal-to-noise ratio values are color-coded from 0 (blue) to 25 (red). Figure 3 can be viewed online in color at www.investigativeradiology.com.

dielectric pads yielded significantly better visibility of anatomic details of articular disc components (anterior band, intermediate zone, and posterior band) as well as bilaminar zone, fossa, condyle, and pterygoid muscle $(P<0.05$, corrected for multiple comparisons, see Fig. 4 and Table 1 for presentation of between-group differences). Moreover, there was no statistically significant difference in the visibility of these TMJ subregions between females and males when using the dielectric pads (see Table 2, $P<0.05$, corrected for multiple comparisons). When using dielectric pads, the left side showed a statistical trend toward better overall image quality $(P=0.046$, uncorrected $)$ as well as better visibility of the intermediate zone of the temporomandibular disc $(P=0.034$, uncorrected) and the bilaminar zone ( $P=0.014$, uncorrected $)$. However, these results did not remain significant after correction for multiple comparisons (see Table 3 for detailed presentation).

\section{DISCUSSION}

A key finding of this study was that dielectric pads provide higher local B1+ and SNR as well as better visibility of anatomical structures when MRI of the TMJ were obtained at 7.0 T. For this purpose, we assessed quantitative data in terms of B1+ maps and SNR maps as well as qualitative data in asymptomatic subjects with and without dielectric pads. Therefore, the present results provide first evidence for the feasibility of imaging the TMJ at 7.0 T using high-permittivity dielectric pads despite the inhomogeneous local $\mathrm{B} 1+$ field distribution.

\section{High-Permittivity Dielectric Pads}

The application of pads consisting of dielectric materials for improving local B1+ fields has been demonstrated in previous studies. ${ }^{19,20}$ In particular, specifically tailored high-permittivity dielectric pads consisting of barium titanate have been used to compensate for the strong $\mathrm{B} 1+$ dropout in the area surrounding the temporal bone by increasing the local B1+ field at cost of the global B1+ field. ${ }^{21}$

In the context of the present study, this effect has been utilized to improve the local B1+ field in the area overlapping with the TMJ. This approach yielded increased SNR in the corresponding area as well as better visibility of the TMJ and its different anatomical structures.

To ensure a standardized approach, care was taken to center both dielectric pads on the TMJs of each subject because improper pad placement might diminish their effectiveness. Another issue that we considered important was sex difference in head size. For clarification, pads were tailored for females and males. However, in this group, pad size in relation to sex revealed no significant difference with respect to SNR and visibility of the TMJ. It is important to note that pads were designed asymmetrical. Based on extensive simulations by another group,${ }^{14}$ this design was found to be best fitted for obtaining a symmetric local $\mathrm{B} 1+$ distribution given the intrinsic asymmetry of $\mathrm{B} 1+$ in the lateral areas of the head. In the current study, SNR was similar between the left and the right TMJ. However, the left side showed in the qualitative analysis a trend toward better overall image quality and visibility of the intermediate zone of the temporomandibular disc as well as the bilaminar zone. Although these results yielded no statistical significance after correction for multiple comparisons, they might represent

TABLE 1. Visibility of Different Anatomical Structures of the Temporomandibular Joint at 7.0 T for Images Acquired With and Without Dielectric Pads and Corresponding Between-Group Differences

\begin{tabular}{|c|c|c|c|c|}
\hline \multirow[b]{2}{*}{$\begin{array}{l}\text { Anatomic } \\
\text { Structure }\end{array}$} & \multirow{2}{*}{$\begin{array}{c}\text { Pads } \\
\text { Mean } \\
\text { (SD) }\end{array}$} & \multirow{2}{*}{$\begin{array}{c}\text { No Pads } \\
\text { Mean } \\
\text { (SD) }\end{array}$} & \multicolumn{2}{|c|}{ Pads vs No Pads } \\
\hline & & & $\begin{array}{c}P \\
\text { (Uncorrected) }\end{array}$ & $\begin{array}{c}P \\
\text { (Corrected) }\end{array}$ \\
\hline \multicolumn{5}{|c|}{ Temporomandibular disc } \\
\hline Anterior band & $1.55(0.75)$ & $4.15(0.87)$ & 0.000073 & 0.000584 \\
\hline Intermediate zone & $1.6(0.68)$ & $4.25(0.78)$ & 0.000068 & 0.000544 \\
\hline Posterior band & $1.5(0.68)$ & $4.2(0.89)$ & 0.000075 & 0.000600 \\
\hline Bilaminar zone & $1.6(0.59)$ & $4.5(0.82)$ & 0.000057 & 0.000456 \\
\hline Mandibular fossa & $1.2(0.41)$ & $4.15(0.74)$ & 0.000056 & 0.000448 \\
\hline Mandibular condyle & $1.2(0.41)$ & $4.15(0.74)$ & 0.000056 & 0.000448 \\
\hline $\begin{array}{l}\text { Inferior pterygoid } \\
\text { muscle }\end{array}$ & $1.65(0.67)$ & $4.15(0.87)$ & 0.000071 & 0.000568 \\
\hline $\begin{array}{l}\text { Overall image } \\
\text { quality }\end{array}$ & $1.4(0.5)$ & $4.25(0.78)$ & 0.000043 & 0.000344 \\
\hline
\end{tabular}

Two fellowship-trained radiologists rated the visibility of each anatomical structure in consensus. Mean (SD) values are given for the visibility of each anatomical structure. Grading was based on a Likert scale from 1 to 5 (1, excellent visibility; 2 , good visibility; 3 , moderate visibility; 4 , poor visibility; 5 , not visible). To evaluate potential differences between the images acquired with and without pads with respect to the visibility of clinically relevant anatomical structures of the temporomandibular joint, Wilcoxon signed rank tests were performed for each structure, respectively. $P$ values are given uncorrected as well as corrected for multiple comparisons $(\mathrm{n}=8)$.

Italic data indicates $p<0.05$. 
With Pads

(1)



A

\section{Without Pads}

(1)

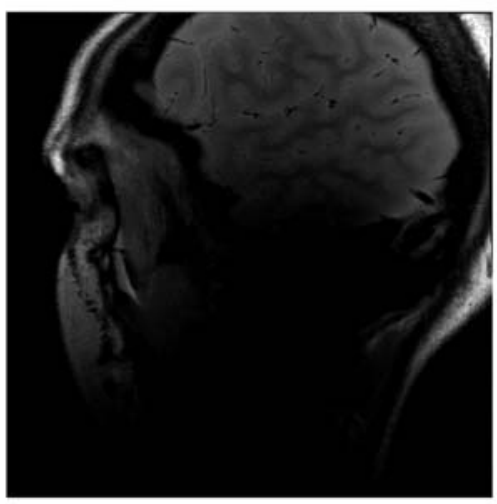

(2)

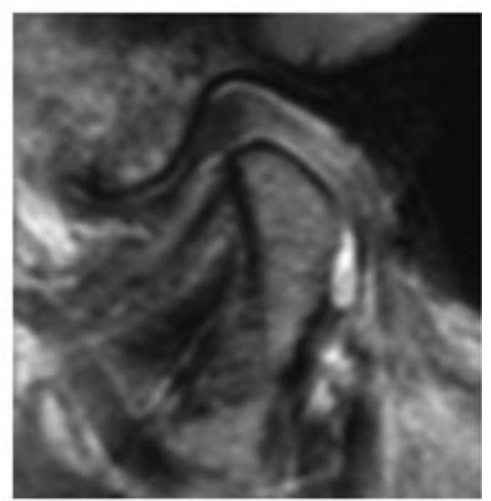

(2)

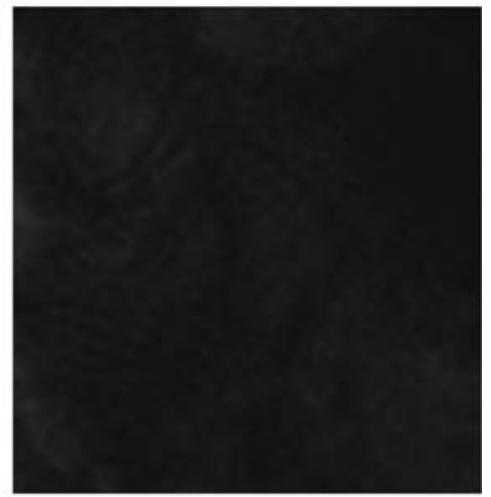

B

FIGURE 4. Qualitative analysis. Proton density-weighted oblique sagittal images in closed mouth position at $7.0 \mathrm{~T}$ acquired with and without dielectric pads. A-1, The image of a temporomandibular joint of an asymptomatic volunteer assessed using dielectric pads. A-2, The corresponding zoomed inset after manual contrast adjustment. B-1, The image of the same TMJ of the same volunteer assessed without dielectric pads. B-2, The corresponding zoomed inset after manual image contrast adjustment. For all subregions of the TMJ, visibility was higher when using dielectric pads.

a hint that additional optimization of the geometrical design (including size and shape) and/or permittivity of the dielectric pads (ie, concentration and composition of dielectric materials) could be beneficial for further improving the effect of dielectric pads on local B1+ fields in the area of the TMJ.

Other technical aspects influencing MRI quality are related to local B1+ fields. According to the literature, improvement can be achieved by using static B1+ shimming or specifically designed RF pulses, which can compensate for B1+ inhomogeneity in combination with dedicated transmit coil arrays. ${ }^{22}$ First, static B1+ shimming has been proposed to adjust the phases of multiple transmit channels to improve the maximum B1+ efficiency, allowing for increased B1+ homogeneity in potential regions of interest. ${ }^{23}$ Recently, several studies demonstrated the successful application of B1+ shimming in different anatomical regions, including the head, ${ }^{23}$ hip joints, ${ }^{24}$ and for renal angiography. ${ }^{25}$ In particular, De Martino et $\mathrm{al}^{23}$ reported that by combining a multichannel transmit RF coil with B1+ shimming methods, MRI could be performed in the bilateral auditory cortex at high quality without exceeding the SAR limits at 7.0 T. Due to its high potential, $\mathrm{B} 1+$ shimming is a promising approach, but requires further studies to investigate its potential benefits, particularly with respect to MRI of the TMJ at 7.0 T. Second, several RF design approaches have been proposed to account for inhomogeneous flip angle distribution, including adiabatic pulses ${ }^{26}$ and spatially tailored excitation designs. ${ }^{27}$ Although both approaches yield a great potential regarding the improvement of the excitation profile at $7.0 \mathrm{~T},{ }^{22,28,29}$ substantial challenges, particularly regarding the SAR management, are still limiting their potential use. ${ }^{14}$

In contrast, dielectric pads are able to enhance local B1+ without increasing the local SAR, ${ }^{13}$ which is important for their application in the clinical routine. Furthermore, they can be utilized independently of experimental setups, including used software routines or hardware platform. Therefore, the application of dielectric pads is an easy and cost-effective way to compensate for low B1+ fields at ultrahigh field strengths. Future studies evaluating the combination of dielectric pads with dedicated RF pulses yield great potential and will be the focus of our next research in this field.

\section{Quantitative and Qualitative Results}

In the current study, SNR has been measured based on a voxelwise approach by repeating the imaging sequences without RF excitations and gradient switching. This procedure accounts for possible noise correlations between individual coil channels and yielded voxelwise SNR maps of the entire FOV, finally resulting in voxelwise maps for the desired region of interest. Applying this method, we found that SNR in the area covering the TMJ was approximately 2 -fold when applying the dielectric pads, suggesting that increased local B1+ translates into increased local SNR. However, it is to note that even with dielectric pads, only $\sim 60 \%$ (ie, an increase of $\sim 15 \%$, see Fig. $2 \mathrm{C}$ ) of the nominal flip angle is achieved in the lateral areas of the head at cost of lower $\mathrm{B} 1+$ in the central regions of the head, which is in line with previous 
TABLE 2. Visibility of Different Anatomical Structures of the Temporomandibular Joint at 7.0 T for Images Acquired With Dielectric Pads for Women and Men and Corresponding Between-Group Differences

\begin{tabular}{|c|c|c|c|c|}
\hline \multirow[b]{2}{*}{$\begin{array}{l}\text { Anatomic } \\
\text { Structure }\end{array}$} & \multirow{2}{*}{$\begin{array}{c}\text { Women } \\
\text { Mean } \\
\text { (SD) }\end{array}$} & \multirow{2}{*}{$\begin{array}{l}\text { Men } \\
\text { Mean } \\
(\text { SD) }\end{array}$} & \multicolumn{2}{|c|}{ Women vs Men } \\
\hline & & & $\begin{array}{c}P \\
\text { (Uncorrected) }\end{array}$ & $\begin{array}{c}P \\
\text { (Corrected }\end{array}$ \\
\hline \multicolumn{5}{|c|}{ Temporomandibular disc } \\
\hline Anterior band & $1.30(0.48)$ & $1.80(0.91)$ & 0.211 & 1.000 \\
\hline Intermediate zone & $1.40(0.51)$ & $1.80(0.78)$ & 0.240 & 1.000 \\
\hline Posterior band & $1.30(0.48)$ & $1.70(0.82)$ & 0.259 & 1.000 \\
\hline Bilaminar zone & $1.50(0.52)$ & $1.70(0.67)$ & 0.522 & 1.000 \\
\hline Mandibular fossa & $1.20(0.42)$ & $1.20(0.42)$ & 1.000 & 1.000 \\
\hline Mandibular condyle & $1.20(0.42)$ & $1.20(0.42)$ & 1.000 & 1.000 \\
\hline $\begin{array}{l}\text { Inferior pterygoid } \\
\text { muscle }\end{array}$ & $1.40(0.51)$ & $1.90(0.73)$ & 0.112 & 0.896 \\
\hline $\begin{array}{l}\text { Overall image } \\
\text { quality }\end{array}$ & $1.20(0.42)$ & $1.60(0.51)$ & 0.075 & 0.900 \\
\hline
\end{tabular}

To account for differences in head size, 2 different sets of dielectric pads were used for female and male volunteers. Two fellowship-trained radiologists rated the visibility of each anatomical structure in consensus. Mean (SD) values are given for the visibility of each anatomical structure. Grading was based on a Likert scale from 1 to 5 (1, excellent visibility; 2, good visibility; 3 , moderate visibility; 4 , poor visibility; 5 , not visible). To evaluate potential between group differences, Mann-Whitney $U$ tests were performed for each structure, respectively. $P$ values are given uncorrected as well as corrected for multiple comparisons $(\mathrm{n}=8)$.

observations. ${ }^{14}$ This residual undertipping might diminish the obtained contrast and cause spurious artifacts due to incomplete refocusing of the transverse magnetization. ${ }^{30}$ Furthermore, high-permittivity dielectric pads are additionally altering the reception fields (B1-), which potentially also modulate the measured SNR. ${ }^{31}$ Since B1- fields have not been assessed in the current study, it was not possible to determine the contribution of potentially increased B1- fields on the increased SNR when using dielectric pads.

Generally, qualitative analysis is commonly performed by 2 independent observers to test for the robustness of results and to account for potential observer bias. However, to the best of our knowledge, this was the first study on 7.0 T imaging of the TMJ. Therefore, rating in consensus was preferred in this study to account for the lack of experience regarding the evaluation of TMJ details at this ultrahigh field strength. Qualitative analysis yielded improved visualization of the anatomical subregions of the TMJ, including articular disc, condyle, fossa, and lateral pterygoid muscle. In particular, the detailed visibility of the articular disc is of great potential because it can currently not be depicted in full detail using lower magnetic field strengths, such as 1.5 or $3.0 \mathrm{~T}$. This might facilitate image segmentation commonly applied in 3-day analysis of the TMJ and the disc in particular, thus improving assessment of TMJ loading. ${ }^{32}$ Finally, a highly detailed depiction of TMJ structures might provide clinicians with additional information for more accurate diagnoses. Although the presented results are promising, further studies comparing imaging of the TMJ at 7.0 T and lower field strengths, such as $3.0 \mathrm{~T}$, are necessary to better appreciate a potential clinical value

\section{Limitations}

The current study has several imitations. The first imitation is the sample size. We included only 10 asymptomatic volunteers, resulting in $20 \mathrm{TMJ}$ images. However, the size of the assessed group is in line with previously reported feasibility studies on TMJ imaging. ${ }^{11,14}$ The second limitation is the pad geometry design. The pads used were originally manufactured based on simulations for imaging the inner ear at 7.0 T. Possibly, SNR and visibility of the TMJ could have been even better if pads had been specifically tailored for the TMJ. However, the pads have previously been shown to improve local B1+ in the temporal bone and adjacent areas, including the areas overlapping with the TMJ. The third limitation is the statistical analysis of B1+. Pad influence on local $\mathrm{B} 1+$ has already been demonstrated in full detail elsewhere, ${ }^{14}$ which is why pad effects are presented here for 1 representative subject only. The fourth limitation is the coil. In the current study, a 32-channel head coil was used. Although the used head coil covered the TMJ in full extent, a recent study evaluating a specific manufactured opthalmic surface coil for imaging the eye at 7.0 $\mathrm{T}$ suggests that the use of close fitting transmit/receive coils might further enhance the visibility of fine anatomical structures at 7.0 $\mathrm{T}^{33}$ Further studies are necessary to assess whether imaging the TMJ at 7.0 T might benefit from such coil designs to the same extent. The fifth limitation is the spatial resolution. It is to note that the applied scan protocol included a voxel size, which is only marginally smaller that in a previous reported study at $3.0 \mathrm{~T},{ }^{8}$ which is mainly explained by the exploratory nature of the current feasibility study. Current findings strongly suggest that the resolution could be further optimized in future studies, potentially yielding even higher visibility of the evaluated anatomical structures. The sixth limitation is the lack of criterion standard. The lack of a criterion standard is a general problem in in vivo MRI. Surgical and/or autoptic reinvestigations of the imaged structures would be required to prove the fidelity of imaging results. To this regard, Sanal and colleagues ${ }^{34}$ demonstrated recently in a postmortem study that imaging the TMJ at 3.0 T provides an adequate characterization of the TMJ's anatomy. This indicates the validity of MRI of the TMJ as an accurate investigational in vivo tool for research and diagnostic depiction of TMJ structures. Nevertheless, further optimization of current MR methods is desirable to ensure a more detailed depiction of the TMJ.

TABLE 3. Visibility of Different Anatomical Structures of the Temporomandibular Joint at 7.0 T for the Left and the Right Side With Dielectric Pads and Corresponding Between-Group Differences

\begin{tabular}{|c|c|c|c|c|}
\hline & Right Side & Left Side & & \\
\hline $\begin{array}{l}\text { Anatomic } \\
\text { Structure }\end{array}$ & $\begin{array}{l}\text { Mean } \\
(\text { SD) }\end{array}$ & $\begin{array}{l}\text { Mean } \\
(\text { SD) }\end{array}$ & $\begin{array}{c}P \\
\text { (Uncorrected) }\end{array}$ & $\begin{array}{c}P \\
\text { (Corrected }\end{array}$ \\
\hline \multicolumn{5}{|c|}{ Temporomandibular disc } \\
\hline Anterior band & $1.90(0.876)$ & $1.20(0.422)$ & 0.059 & 0.470 \\
\hline $\begin{array}{l}\text { Intermediate } \\
\text { zone }\end{array}$ & $1.90(0.738)$ & $1.30(0.483)$ & 0.034 & 0.271 \\
\hline Posterior band & $1.80(0.789)$ & $1.20(0.422)$ & 0.063 & 0.507 \\
\hline Bilaminar zone & $1.90(0.568)$ & $1.30(0.483)$ & 0.014 & 0.114 \\
\hline Mandibular fossa & $1.30(0.483)$ & $1.10(0.316)$ & 0.317 & 1.000 \\
\hline $\begin{array}{l}\text { Mandibular } \\
\text { condyle }\end{array}$ & $1.30(0.483)$ & $1.10(0.316)$ & 0.157 & 1.000 \\
\hline $\begin{array}{l}\text { Inferior pterygoid } \\
\text { muscle }\end{array}$ & $1.90(0.568)$ & $1.40(0.699)$ & 0.059 & 0.470 \\
\hline $\begin{array}{l}\text { Overall image } \\
\text { quality }\end{array}$ & $1.60(0.516)$ & $1.20(0.422)$ & 0.046 & 0.364 \\
\hline
\end{tabular}

To account for the intrinsic asymmetry of B1+ in the lateral regions of the head, pads were specifically designed for the left and the right side. Two fellowship-trained radiologists rated the visibility of each anatomical structure in consensus. Mean (SD) values are given for the visibility of each anatomical structure. Grading was based on a Likert scale from 1 to 5 (1, excellent visibility; 2 , good visibility; 3 , moderate visibility; 4 , poor visibility; 5 , not visible). To evaluate potential between group differences, Wilcoxon signed rank tests were performed for each structure, respectively. $P$ values are given uncorrected as well as corrected for multiple comparisons $(\mathrm{n}=8)$. 


\section{Clinical Implications}

Current imaging approaches do not provide the necessary spatial resolution to depict anatomical substructures of the TMJ such as the articular disc in full detail, possibly explaining the lack of correlation between clinical symptoms and imaging findings. ${ }^{10}$ Because the SNR is assumed to increase almost linearly with static magnetic field strength, gains in SNR at 7.0 T can be used to increase the spatial resolution. This provides a more adequate depiction of the TMJ and may result in improved diagnostic sensitivity. However, the scope of the current study was restricted to the evaluation of the feasibility of imaging the TMJ at 7.0 T. Future studies comparing imaging the TMJ at 7.0 T with clinical standard protocols at lower field strengths are needed to evaluate the potential benefits for the diagnostic process and the potential impact on clinical decision making when MRI patients with TMDs.

\section{CONCLUSIONS}

High-permittivity dielectric pads improve local B1+ and SNR of the TMJ, which translates into better visibility of the TMJ at 7.0 T. These results demonstrate that imaging the TMJ at 7.0 $\mathrm{T}$ is feasible when using dielectric pads.

\section{ACKNOWLEDGMENTS}

The authors are grateful to Andrew G. Webb and Wyger M. Brink for providing the high-permittivity dielectric pads, Stefan Erni for his thorough introduction into the practical aspects of TMJ imaging, Erika J. Ulbrich for her valuable advice regarding the study design, Constantin von Deuster for his help in visualizing the results, as well as Leatitia Vionnet, Andre Kuehne and Ariane Fillmer for many helpful discussions on the theoretical aspects of B1+field inhomogeneities.

\section{REFERENCES}

1. McNeill C. Management of temporomandibular disorders: concepts and controversies. J Prosthet Dent. 1997;77:510-522.

2. Weinberg LA. The etiology, diagnosis, and treatment of TMJ dysfunction-pain syndrome. Part I: etiology. J Prosthet Dent. 1979;42:654-664.

3. De Rossi SS. Orofacial pain: a primer. Dent Clin North Am. 2013;57:383-392.

4. White BA, Williams LA, Leben JR. Health care utilization and cost among health maintenance organization members with temporomandibular disorders. J Orofac Pain. 2001;15:158-169.

5. Manfredini D, Guarda-Nardini L, Winocur E, et al. Research diagnostic criteria for temporomandibular disorders: a systematic review of axis I epidemiologic findings. Oral Surg Oral Med Oral Pathol Oral Radiol Endod. 2011;112:453-462.

6. Ogutcen-Toller M, Taskaya-Yilmaz N, Yilmaz F. The evaluation of temporomandibular joint disc position in TMJ disorders using MRI. Int J Oral Maxillofac Surg. 2002;31:603-607.

7. Schmid-Schwap M, Drahanowsky W, Bristela M, et al. Diagnosis of temporomandibular dysfunction syndrome-image quality at 1.5 and 3.0 Tesla magnetic resonance imaging. Eur Radiol. 2009;19:1239-1245.

8. Stehling C, Vieth V, Bachmann R, et al. High-resolution magnetic resonance imaging of the temporomandibular joint: image quality at 1.5 and 3.0 Tesla in volunteers. Invest Radiol. 2007;42:428-434.

9. Iwasaki H, Kubo H, Harada M, et al. Temporomandibular joint and $3.0 \mathrm{~T}$ pseudodynamic magnetic resonance imaging. Part 1: evaluation of condylar and disc dysfunction. Dentomaxillofac Radiol. 2010;39:475-485

10. Koh KJ, List T, Petersson A, et al. Relationship between clinical and magnetic resonance imaging diagnoses and findings in degenerative and inflammatory temporomandibular joint diseases: a systematic literature review. J Orofac Pain. 2009; 23:123-139.

11. Nordmeyer-Massner JA, Wyss M, Andreisek G, et al. In vitro and in vivo comparison of wrist MR imaging at 3.0 and 7.0 tesla using a gradient echo sequence and identical eight-channel coil array designs. J Magn Reson Imaging. 2011;33: 661-667.

12. Hsu YC, Chern IL, Zhao W, et al. Mitigate B1+ inhomogeneity using spatially selective radiofrequency excitation with generalized spatial encoding magnetic fields. Magn Reson Med. 2014;71:1458-1469.

13. Haines K, Smith NB, Webb AG. New high dielectric constant materials for tailoring the B1+ distribution at high magnetic fields. J Magn Reson. 2010;203 323-327.

14. Brink WM, van der Jagt AM, Versluis MJ, et al. High permittivity dielectric pads improve high spatial resolution magnetic resonance imaging of the inner ear at $7 \mathrm{~T}$. Invest Radiol. 2014;49:271-277.

15. Yarnykh VL. Actual flip-angle imaging in the pulsed steady state: a method for rapid three-dimensional mapping of the transmitted radiofrequency field. Magn Reson Med. 2007;57:192-200.

16. Nordmeyer-Massner JA, De Zanche N, Pruessmann KP. Mechanically adjustable coil array for wrist MRI. Magn Reson Med. 2009;61:429-438.

17. Roemer PB, Edelstein WA, Hayes CE, et al. The NMR phased array. Magn Reson Med. 1990;16:192-225.

18. Pruessmann KP, Weiger M, Scheidegger MB, et al. Sense: sensitivity encoding for fast MRI. Magn Reson Med. 1999;42:952-962.

19. Snaar JE, Teeuwisse WM, Versluis MJ, et al. Improvements in high-field localized MRS of the medial temporal lobe in humans using new deformable high-dielectric materials. NMR Biomed. 2011;24:873-879.

20. Teeuwisse WM, Brink WM, Haines KN, et al. Simulations of high permittivity materials for $7 \mathrm{~T}$ neuroimaging and evaluation of a new barium titanate-based dielectric. Magn Reson Med. 2012;67:912-918.

21. Teeuwisse WM, Brink WM, Webb AG. Quantitative assessment of the effects of high-permittivity pads in 7 Tesla MRI of the brain. Magn Reson Med. 2012;67: 1285-1293.

22. Setsompop K, Alagappan V, Gagoski B, et al. Slice-selective rf pulses for in vivo $\mathrm{B} 1+$ inhomogeneity mitigation at 7 Tesla using parallel RF excitation with a 16-element coil. Magn Reson Med. 2008;60:1422-1432.

23. De Martino F, Schmitter S, Moerel M, et al. Spin echo functional MRI in bilateral auditory cortices at $7 \mathrm{~T}$ : an application of $\mathrm{B}_{1}$ shimming. Neuroimage. 2012;63 1313-1320.

24. Ellermann J, Goerke U, Morgan P, et al. Simultaneous bilateral hip joint imaging at 7 Tesla using fast transmit $\mathrm{B}_{1}$ shimming methods and multichannel transmission — a feasibility study. NMR Biomed. 2012;25:1202-1208.

25. Metzger GJ, Auerbach EJ, Akgun C, et al. Dynamically applied B1+ shimming solutions for non-contrast enhanced renal angiography at 7.0 Tesla. Magn Reson Med. 2013;69:114-126.

26. Garwood M, DelaBarre L. The return of the frequency sweep: designing adiabatic pulses for contemporary NMR. J Magn Reson. 2001;153:155-177.

27. Saekho S, Boada FE, Noll DC, et al. Small tip angle three-dimensional tailored radiofrequency slab-select pulse for reduced B1 inhomogeneity at 3 T. Magn Reson Med. 2005;53:479-484.

28. Grissom WA, Khalighi MM, Sacolick LI, et al. Small-tip-angle spokes pulse design using interleaved greedy and local optimization methods. Magn Reson Med. 2012;68:1553-1562

29. Setsompop K, Wald LL, Alagappan V, et al. Magnitude least squares optimization for parallel radio frequency excitation design demonstrated At 7 Tesla with eight channels. Magn Reson Med. 2008;59:908-915.

30. Weigel M. Extended phase graphs: dephasing, RF pulses, and echoes - pure and simple. J Magn Reson Imaging. 2015;41:266-295.

31. Yang QX, Rupprecht S, Luo W, et al. Radiofrequency field enhancement with high dielectric constant (HDC) pads in a receive array coil at 3.0 T. J Magn Reson Imaging. 2013;38:435-440.

32. Ettlin DA, Mang H, Colombo V, et al. Stereometric assessment of TMJ space variation by occlusal splints. J Dent Res. 2008;87:877-881.

33. Graessl A, Muhle M, Schwerter M, et al. Ophthalmic magnetic resonance imaging At 7 T using a 6-channel transceiver radiofrequency coil array in healthy subjects and patients with intraocular masses. Invest Radiol. 2014;49:260-270.

34. Sanal HT, Bae WC, Pauli C, et al. Magnetic resonance imaging of the temporomandibular joint disc: feasibility of novel quantitative magnetic resonance evaluation using histologic and biomechanical reference standards. J Orofac Pain. 2011;25:345-353. 